photographer of country-life, especially tree studies, and here he regales us with some of the best examples of his art. No lover of Nature, no amateur or professional photographer; could fail to be charmed by these studies of country-life taken in all moods and in every season, and through the medium of these photographs the book must appeal to many more than those who merely want to know about Epping Forest. Notwithstanding this, Londoners and others who contemplate visiting that area would do well to consult this book first.

Mr. Brimble (no relative of one of Nature's editors of the same name) is a book-seller, and the present volume is the fruit of his well-spent leisure. Both he and his publishers are to be congratulated on a well-written, superbly illustrated and handsomely produced book.

\section{PRACTICAL PHYSICAL CHEMISTRY}

\section{Physico-Chemical Methods}

By Prof. Joseph Reilly and Prof. William Norman Rae. Vol. 3 (Supplementary). Pp. ix +698 . (London : Methuen and Co., Ltd., 1948.) 55s, net.

THIS book is a supplementary volume to the fourth revised edition of "Reilly and Rae" published in 1943. It contains much material that could not be accommodated in Vols. 1 and 2 and much additional information that has become available as a result of post-war advances in physical chemistry. It may truthfully be said there is no defined limit to physico-chemical methods. On one hand, much that the physicist has developed in other connexions is rapidly adapted and modified for purely chemical purposes; on the other hand, the application of physical chemistry to substances and reactions of biological interest has pushed forward to the extreme the developments of highly complex physical chemical methods. Due regard is paid in this volume to these important borderline activities in modern physical chemistry. From this it becomes evident that no pair of authors can possibly have even a nodding acquaintance with the limitless variety of topics that must find a place in a comprehensive reference book of this kind. A number of chapters are therefore written by other authors who have a special know. ledge of the subjects discussed.

The selection and balance of the topics is a most difficult one, and no two physical chemists would agree on this point. The book opens with a brief account of electron optics as a preliminary to a description of the electron microscope. Few physical chemists would now try to build such a highly specialized instrument; it is therefore a pertinent question as to whether valuable space in this book should properly be devoted to this subject. Similar remarks might be made of the chapter on the mass spectrograph, except that here an indication of its usefulness rather than its construction should now find a place in any physical text-book of an advanced character. The chapter on photo-physics-mainly on photocells:-is extremely useful and could be expanded with advantage in a future edition, for chemists are not always aware of what can be done with these tools. The determination of atomic and molecular weights occupies a considerable portion, due emphasis being paid to micro methods for molecular weight determination. Osmotic pressure apparatus is adequately dealt with, and more information about modern instruments for measuring very small pressures would be welcome. The procedure, rather than the apparatus, for dealing with homogeneous reaction velocities is set out in detail so that effective use may be made of the more practical procedures described in other parts of the volumes. Chromatography properly gets its due quota of space, and the theory is properly considered so that the approach to it need not be purely empirical. The preparation of colloidal solutions perhaps occupies a rather large amount of space, since the quantitative significance of the subject is never particularly high. There are many more topics, but lack of space prevents their being mentioned.

The great value of a work of this kind is as a source book for guidance-it cannot be critical, for the subject is too vast. What it can and does do is to provide the reader with ideas, methods of approach and devices to help him in his particular problems in this field of chemistry.

H. W. Melvirite

\section{GENERAL INTRODUCTION TO ADVANCED MAGNETISM}

\section{Magnetism}

By Dr. David Shoenberg. (Sigma Introductions to Science, 12.) Pp. $x+216+8$ plates. (London : Sigma Books, Ltd., 1949.) 10s. 6d. net.

$7 \mathrm{O}$ give a satisfying account of magnetism in some two hundred pages is a considerable undertaking; to accomplish this as excellently as he has done, Dr. Shoenberg must have given a great deal of thought to the impact of quantitative ideas and conventions on the general reader meeting them for the first time. In the early chapters, the single magnetic pole is soon discarded for the dipole, and the relation between magnetism, electricity and atomic structure is pursued via magnetic shells to electron orbits and the ultimate spin. None of the essential theory of elementary magnetism is omitted, but it appears in a guise less formidable and more convincing than usual.

The account of magnetic materials and ferromagnetics is valuable from two points of view : the domain-structure idea and some evidence for it are well explained ; and there is also some quantitative discussion of such points as the importance of coercivity rather than merely remanence for a permanent-magnet steel, and what happens when a keeper is removed and replaced again. There are chapters on terrestrial magnetism, the use of magnetism as a tool in scientific work, and some everyday applications of it. These are not very detailed, but the book is professedly an introduction and lives up to this by giving a list of standard works in which the topics may be pursued further. The glossaryindex combination is a useful idea. The diagrams are good, but the eight plates would have brightened the volume more if they had been spaced out instead of being centralized.

The author is lecturer in physics in the University of Cambridge and head of the Royal Society Mond Laboratory at Cambridge, having been there since 1932. As the publishers state, the book is of a more advanced character than most of the Sigma series, and it should be useful to the serious student as well as to the general reader, both of whom will appreciate in its pages the authentic hand of the expert.

G. R. NOAKES 